\title{
Experiment for Non-Interfering Coexistence of Non-Orthogonal SEFDM Signals and LTE
}

\author{
Tongyang $\mathrm{Xu}$ and Izzat Darwazeh \\ Department of Electronic and Electrical Engineering, University College London, London, UK \\ Email: t.xu@ee.ucl.ac.uk, i.darwazeh@ucl.ac.uk
}

\begin{abstract}
A non-orthogonal waveform, termed Nyquistspectrally efficient frequency division multiplexing (NyquistSEFDM), introduces benefits such as compressed bandwidth and low out-of-band power leakage, which meets two crucial requirements for future carrier aggregation (CA) or machinetype communication (MTC) applications. Both simulation and experimental results are presented in this work. A comparative simulation study of SEFDM signals is firstly presented to show the trade-offs between BER performance, bandwidth compression and suppressed out-of-band power leakage. Following modelling results, an enhanced multiuser diversity scenario was proposed to show future applications of SEFDM waveforms. Finally, an experimental testbed was designed to validate the coexistence of two signals. The impact of the newly proposed signals on existing $4^{t h}$ generation (4G) signals is evaluated.

Index Terms-Multicarrier, experiment, testbed, software defined radio, pulse shaping, spectral efficiency, carrier aggregation, OFDM, SEFDM, non-orthogonal.
\end{abstract}

\section{INTRODUCTION}

Multicarrier waveforms are widely used in modern communication systems due to their high immunity to multipath fading. OFDM is the standardized waveform in LTE [1] and LTE-Advanced [2]. For future $5^{\text {th }}$ generation $(5 \mathrm{G})$ networks [3], data rate is not the only requirement; reliable services for large number of connected devices are becoming more important in internet of things (IoT) [4], [5] and MTC [6] scenarios. Unfortunately, current deployed OFDM waveforms create large sidelobes and therefore interference to adjacent signals.

Work in [7] proposed a waveform termed spectrally efficient frequency division multiplexing (SEFDM), which compresses bandwidth by packing sub-carriers closer. It was further extended to multi-antenna systems in [8] where capacity was studied. Spectral efficiency is improved leading to an improved data rate. The experimental work in [9] verified that more signal bands can be packed without occupying extra bandwidth resources. It would be beneficial to MTC scenarios. However, frequency protection gaps are still needed due to the high out-of-band power leakage. Therefore, work in [10] proposed an improved waveform termed NyquistSEFDM, which can compress bandwidth and suppress outof-band power leakage, simultaneously. This novel waveform enables non-interfering coexistence between multiple signal bands via shaping the modulating symbols on each sub-carrier. It has been shown that $20 \mathrm{~dB}$ out-of-band power reduction, compared to typical OFDM waveforms, is achievable.
This work extends the theoretical work in [10] by studying adjacent and non-adjacent sub-carrier interference and their effects on bit error rate (BER) performance. Furthermore, this work designed an experimental testbed for validations of coexistent signals, which can be used to show the benefits of Nyquist-SEFDM signals in MTC scenarios in future 5G systems. In the experiment section, two signals are aggregated. The first signal is the long term evolution (LTE) OFDM signal and the second signal can be flexibly modified in order to study different effects on the first signal. The performance of the first signal, interfered by the second signal, is evaluated via considering of constellation diagrams.

\section{WAVEFORM DESIGN}

The non-orthogonal SEFDM signal has been systematically studied in previous work [9]. Its discrete signal of $N$ subcarriers is expressed as

$$
X[k]=\frac{1}{\sqrt{Q}} \sum_{n=0}^{N-1} s_{n} \exp \left(\frac{j 2 \pi n k \alpha}{Q}\right)
$$

where $X[k]$ is the sample with index $k$ ranging from 0 to $Q-1, Q=\rho N$ and $\rho \geq 1$ is the oversampling factor, $s_{n}$ is the symbol modulated on the $n$th sub-carrier, $\alpha$ is the bandwidth compression factor defined as $\alpha=\Delta f T, T$ indicates one SEFDM symbol duration and $\Delta f$ represents the sub-carrier spacing. The concept of bandwidth compression can be explained by Fig. 1 where two spectra are showing. The sub-carrier spacing of OFDM is $15 \mathrm{kHz}$ while SEFDM shows a narrower spacing $10.8 \mathrm{kHz}$. Sub-carriers in both cases have the same basedband modulation bandwidth of $15 \mathrm{kHz}$. While in OFDM, all sub-carriers have null amplitudes at each sub-carrier peak frequency, in SEFDM signals, adjacent sub-carriers do not cross the null amplitude resulting in interference and due to the sidelobe of the sinc spectrum, non-adjacent sub-carriers also introduce interference to a subcarrier.

One solution to mitigate inter carrier interference (ICI) is to pulse shape modulating symbols on each sub-carrier using root raised cosine (RRC) filter [10]. Therefore, sidelobes can be suppressed. Fig. 2 illustrates three-dimensional plots of interference patterns for various systems. The left hand side of the figure shows interference patterns for OFDM and SEFDM systems with no signal shaping. The right hand side of the figure shows corresponding interference patterns when RRC pulse shaping [10] is used. The numbers on the $\mathrm{X}$-axis 

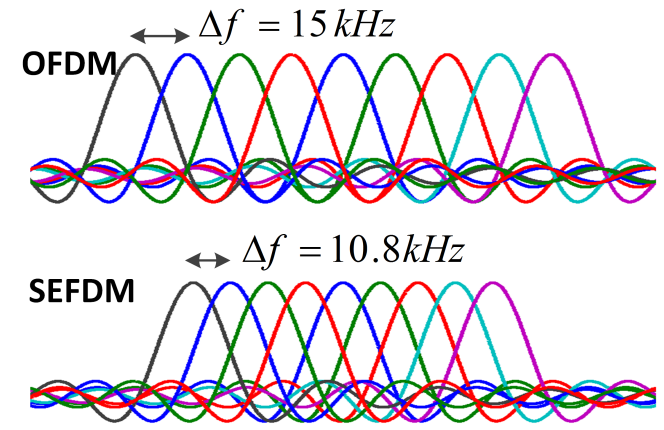

Figure 1. Spectra illustration for OFDM and SEFDM ( $\alpha=0.72)$.

(indexed by $x$ ) and the Y-axis (indexed by $y$ ) correspond to sub-carrier indices. Both $x$ and $y$ scale range from 1 to $N$. The X-Y plane indicates the correlation between sub-carriers where its mathematical explanation can be found in [9]. The numbers on the $\mathrm{Z}$-axis (indexed by $z$ ) indicate absolute values of the correlation coefficients. It should be noted that the auto correlation coefficients are denoted by $I(x, x)$ and the cross correlation coefficients are $I(x, y)$ indicating ICI. For clarity of the figures the number of sub-carriers is limited to $N=16$ and the auto correlation elements are removed (i.e. $I(x, x)=0$ ), thereby only the cross correlation values are shown.
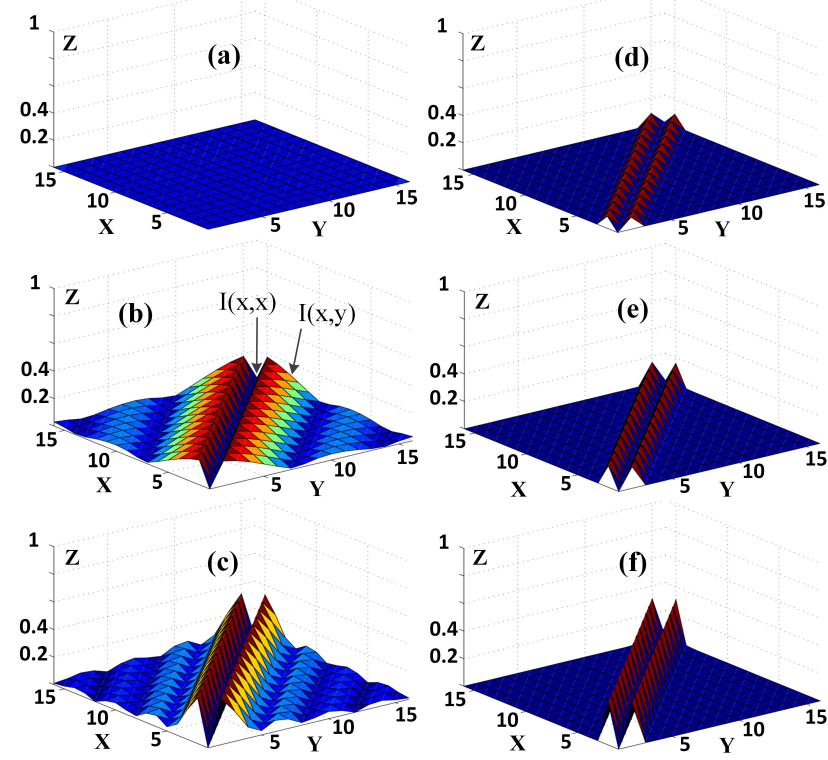

Figure 2. Three-dimensional interference comparison. (a) OFDM; (b) SEFDM $\alpha=0.84$; (c) SEFDM $\alpha=0.72$ and (d) OFDM with RRC shaping; (e) SEFDM $\alpha=0.84$ with RRC shaping; (f) SEFDM $\alpha=0.72$ with RRC shaping.

Fig. 2(a) expectedly shows no interference for the case of OFDM. Fig. 2(b) illustrates a non-shaped SEFDM system with $16 \%$ bandwidth compression $(\alpha=0.84)$. It is apparent that the interference is no longer zero and its values vary depending on frequency distance since sub-carriers are no longer orthogonal. The interference levels clearly vary with the index (i.e frequency location) of the sub-carriers with highest levels evident for adjacent sub-carriers (along the diagonal of the X-Y plane). The interference in a higher bandwidth compressed SEFDM system with $28 \%$ bandwidth compression $(\alpha=0.72)$ is shown in Fig. 2(c), where higher levels are noticed (relative to the two figures above) as the subcarriers become more tightly packed and therefore interfere with higher powers across the overall frequency band. Clearly, further bandwidth compression will result in more interference both from adjacent and non-adjacent sub-carriers.

When RRC pulse shaping is applied to an OFDM system in Fig. 2(d), limited levels of interference appear but only at the two adjacent sub-carriers. This is in contrast to the case of Fig. 2(a) where zero interference appears when no shaping is applied. Comparatively, when applying RRC pulse shaping to SEFDM, interference levels increase in proportion to the bandwidth compression levels as shown in Fig. 2(e) and Fig. 2(f). However, the interference becomes limited only to the adjacent sub-carriers, unlike the non-shaped SEFDM cases of Fig. 2(b) and 2(c), where interference occurs across all the sub-carriers space. Hence, advantageously moving away from the adjacent sub-carriers, interference is eliminated, even for the higher bandwidth compression case of Fig. 2(f).

\section{Filtering And Detection Studies}

This section extends the previous work in [10] to show the effect of adjacent and non-adjacent interference. The simulation parameters follow the same system configurations in [10]. In Fig. 3, both full and partial signal detection are evaluated. The full signal detection indicates that interference is removed from the $N-1$ sub-carriers while the partial signal detection means that only neighbouring interference is removed. The performance is tested at $E_{b} / N_{o}=5 \mathrm{~dB}$. In this work, convolutional coding is used with Turbo equalization [9], [11], noted as scenario B in [10]. Other coding schemes such as Turbo coding [12], [13] and low density parity check (LDPC) coding [14] have also been explored for SEFDM. The basic working principle of the Turbo equalization is to iteratively remove interference from the decoded symbols. After a number of iterations, the performance is fixed with no further improvement even with extra iterations. In Fig. 3(a), for both SEFDM with $\alpha=0.72$ and 0.84 , two iterations are sufficient to get converged performance. In Fig. 3(b), partial signal detection is evaluated for the same systems. One iteration is required for $\alpha=0.72$ SEFDM while two iterations for $\alpha=0.84$ SEFDM. It is clearly seen that the partial-REC SEFDM performance is worse than that of the full-REC SEFDM. Fig. 3(a)(b) indicate that interference to one subcarrier comes not only from neighbouring sub-carriers but also from non-adjacent sub-carriers.

Fig. 3(c)(d) compare different systems in terms of BER performance. In Fig. 3(c), it is apparent that the full-REC detection scheme without pulse shaping shows the best result since all the interference has been removed. When the partialREC detection scheme is applied, error performance suffers several $\mathrm{dBs}$ of degradation. However, when RRC shaping is used, applying the partial detection results in close performance to the one achieved using the full detection without 

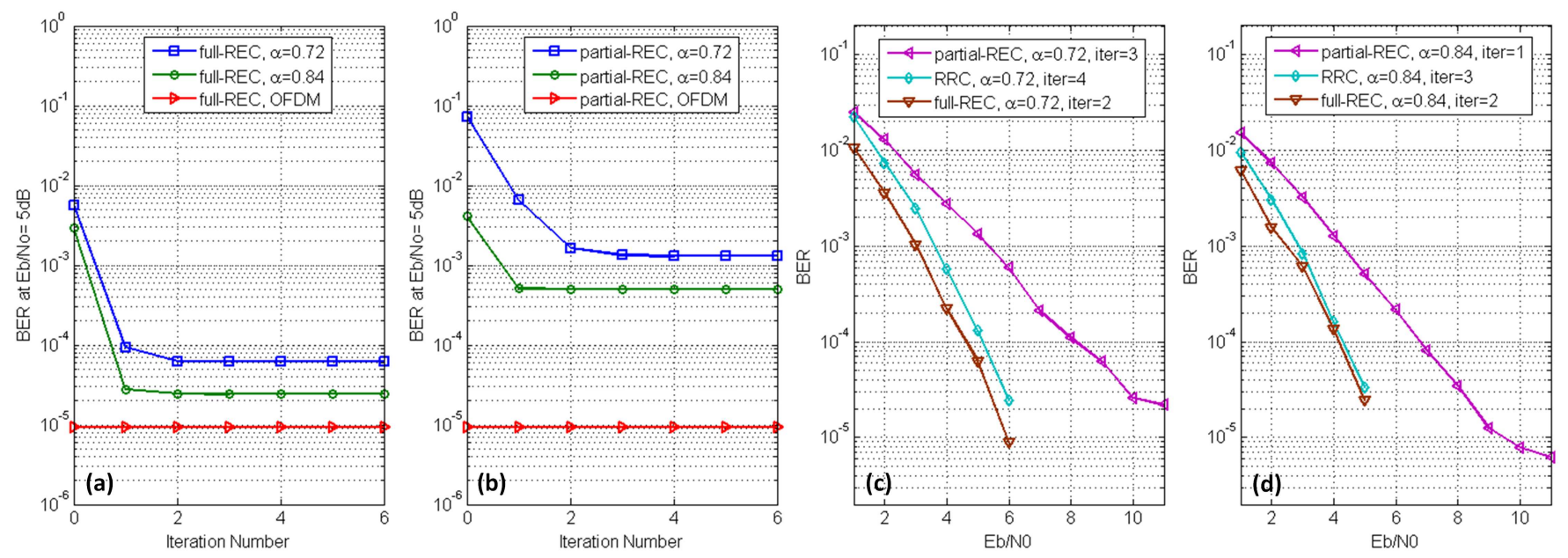

Figure 3. Convergence and BER performance for full-REC (rectangular pulse shaped), partial-REC and RRC (RRC pulse shaped) systems. (a) full-REC; (b) partial-REC; (c) $\alpha=0.72$ and (d) $\alpha=0.84$.

signal shaping. However, more iterations are required for the RRC signal since the extra interference coming from pulse shaping filter has to be removed. The same results are observed for $\alpha=0.84$ in Fig. 3(d). However, due to the relaxed bandwidth compression compared to that in Fig. 3(c), the performance gap is narrowed and the required iteration number to achieve converged performance is reduced.

To sum up, using RRC pulse shaping results in interference limited to adjacent sub-carriers, which can be removed easily at the receiver with minimal performance degradation. It can be seen from Fig. 3(c)(d) that a narrow performance gap exists between the RRC result and the full-REC result. This gap is cased by interference from the uncleared sidelobe due to the truncation effect [10] of pulse shaping filters. In addition, joint effects from bandwidth compression factor, roll-off factor, filter size and the number of detection iterations could affect the final performance, which needs further investigations.

\section{APpliCATION SCENARIO - ENHANCED USER MULTIPLEXING}

In the previous work [9], CA has been explained and experimentally showed that multiple legacy radio bands may be aggregated to support higher data rates. However, typical rectangular signals (i.e. no signal shaping) are specified in LTE-Advanced. The high out-of-band power leakage of one component carriers (CC) poses harmful interference to adjacent CCs. Thus, a $10 \%$ protection gap is inserted between adjacent CCs resulting in an inefficient spectrum utilization. Using the pulse shaping concept in the CA scenario, the outof-band power leakage of a $\mathrm{CC}$ would be reduced. Thus, the $10 \%$ protection gap could be removed leading to further improved spectral efficiency.

As different users in the same frequency band may have different signal-to-noise ratio (SNR) or signal-to-interferenceplus-noise ratio (SINR), it would be more efficient to allow users to select their own preferred subset of sub-carriers with better channel conditions, rather than selecting a single user that occupies all the sub-carriers at the same time. In an OFDM system with a frequency division multiple access (FDMA) scenario termed OFDM-FDMA (OFDMA), users are arranged in both the time and frequency domain. In this case, all users can share the same bandwidth. By using the SEFDM technique, we may introduce an enhanced user multiplexing scheme named SEFDM-FDMA (SEFDMA). Taking into account the CA-OFDMA in a CA scenario, 5 CCs can be allocated to 5 different users at one time. However, in order to provide service to more users, CA-OFDMA has to provide more available bandwidths. In the CA-SEFDMA scheme, we compress each $\mathrm{CC}$ and integrate two more $\mathrm{CCs}$ in a given bandwidth. In other words, 2 more users can be served at one time. In terms of the multiuser diversity, since more CCs are introduced, each $\mathrm{CC}$ with a narrower bandwidth could experience a channel with flatter frequency over the typical one. Our proposal is flexible in a CA scenario since the number of $\mathrm{CCs}$ in a given bandwidth can be dynamically configured based on users' requirements and fading channel conditions.

Fig. 4 illustrates an example that shows how users are scheduled according to fading channel conditions. In order to simplify the demonstration, three users are considered. Their channel states are shown in the figure represented by different frequency responses. The benefit of multiuser diversity is more obvious in a CA-SEFDM scenario since the bandwidth of each band is narrower than a band in OFDM. Therefore, the probability of experiencing fading channel for each SEFDM band is lower than that in OFDM. It is apparent that the radio resources are shared with different users based on different fading conditions. More specifically, depending on the channel SINR, different CCs will be allocated to different users. The channel user 1 experiences is marked with a black line where two deep fading points are observed. In terms of CA-OFDM, only $\mathrm{CC}(2,4,5)$ can be allocated due to their high channel SINR. The CC $(1,3)$ are not used because of the deep fading 


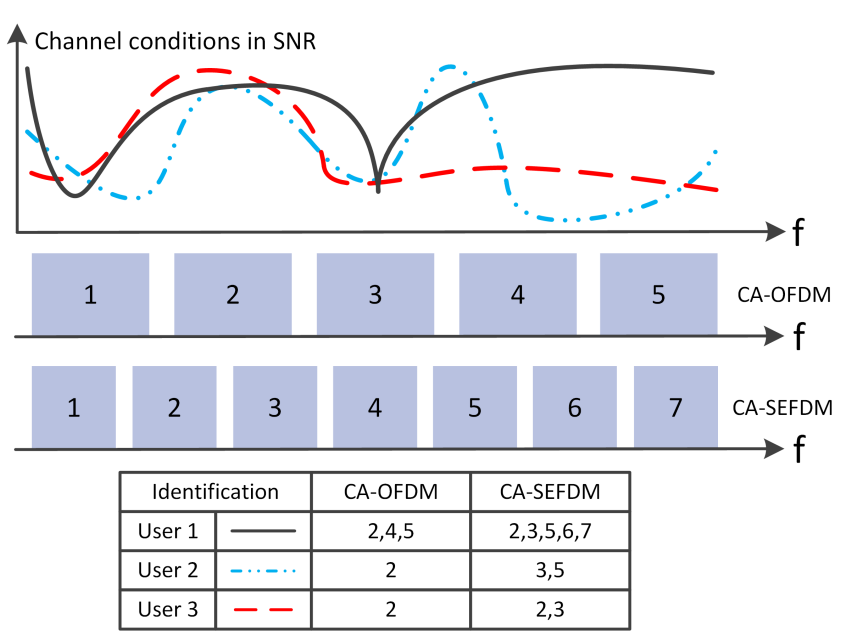

Figure 4. Example of user scheduling according to channel conditions.

effect. On the other hand, for CA-SEFDM, considering the same fading channel condition, $\mathrm{CC}(2,3,5,6,7)$ are available for user 1. It is because that the bandwidth of each $\mathrm{CC}$ is compressed, the effect of deep fading is confined to a small bandwidth CC. There would be more unaffected frequency resources that can be allocated to user 1 . The allocation principle is the same for user 2 and user 3. It is noted that in the CA-OFDM scenario, both user 2 and user 3 are assigned with $\mathrm{CC}(2)$. This indicates only one user (either user 2 or user 3 ) can be served. However, in the case of CA-SEFDM, user 2 is assigned with $\mathrm{CC}(3,5)$ while $\mathrm{CC}(2,3)$ are allocated to user 3 . Therefore, in the CA-SEFDM scenario, user 2 can use CC(5) and user 3 can use $\mathrm{CC}(2)$ indicating that both user 2 and user 3 can be served simultaneously with CC(3) allocated to other users. Based on the above analysis, it is apparent that in the same fading channel condition, CA-SEFDM can support user 1 , user 2 and user 3 while in CA-OFDM only user 1 and user 2 (or user 3) can be served. This indicates that CA-SEFDM has an enhanced multiuser diversity leading to a more flexible multiuser scheduling ability.

\section{EXPERIMENT SETUP}

The coexistence experiment testbed is shown in Fig. 5 where the left inset is a real testbed setup and the right one is a framework illustration. In order to operate the experiment, two Aeroflex PXIs are required. The configurations of the two PXIs are shown in Table I where they have different carrier frequencies. One PXI is used as the signal transmitter for existing LTE OFDM signals and the other one is for the SEFDM. It should be noted that the $5 \mathrm{G}$ testbed configuration is flexible and it is easily reconfigured to another signal standards. The experiment here is to test the out-of-band power effect on existing $4 \mathrm{G}$ signals. Therefore, two signals would be aggregated very close with a $10 \%$ protection gap, as defined for LTE. The main benefit of this testbed is that the entire SEFDM system can be jointly realized in software and hardware. MATLAB is the software tool that implements part of the functions aligning with hardware integrated in the PXI platform. A PC is connected to the PXI and is used to generate digital signals and process received digital signals. Analogue signal processing is integrated in the PXI chassis. Two crucial modules inside the PXI are RF signal generator and RF signal digitizer. The carrier frequency ranges from $100 \mathrm{kHz}$ to $6 \mathrm{GHz}$ on both modules. Up to $90 \mathrm{MHz}$ RF modulation bandwidth and $512 \mathrm{MHz}$ sampling rate are supported. The fixed-point resolution is 32 bit sample word consisting of 14 bit I data, 14 bit $\mathrm{Q}$ data and 4 bit marker data. The detailed information of the front panel of the PXI chassis is provided below.
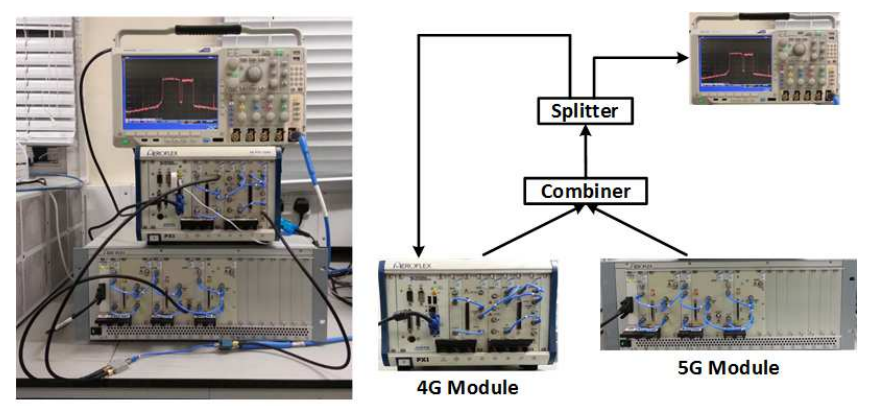

Figure 5. Practical 4G and 5G coexistence testbed setup.

Table I

EXPERIMENTAL TESTBED SPECIFICATIONS

\begin{tabular}{lll}
\hline Parameters & 4G $($ OFDM) & 5G \\
\hline \hline Sampling rate $(\mathrm{MHz})$ & 30.72 & 30.72 \\
\hline Carrier frequency $(\mathrm{GHz})$ & 2 & 2.018 \\
\hline Bandwidth $(\mathrm{MHz})$ & 18 & 15 \\
\hline FFT size & 2048 & 128 \\
\hline Sub-carrier bandwidth $(\mathrm{kHz})$ & 15 & 240 \\
\hline Symbol rate per sub-carrier $(\mathrm{kS} / \mathrm{s})$ & 15 & 240 \\
\hline Sub-carrier spacing $(\mathrm{kHz})$ & 15 & $240 \times \alpha$ \\
\hline Roll-off factor & None & 0.5 \\
\hline
\end{tabular}

The benefit of Nyquist-SEFDM is experimentally demonstrated in Fig. 6 where a non-shaped SEFDM signal and an RRC shaped SEFDM signal are compared. The bandwidth compression factor is set to $\alpha=0.72$, which is derived from previous work [9]. It is apparent that the RRC pulse shaped SEFDM signal outperforms the non-shaped SEFDM signal in terms of the out-of-band power leakage by over $20 \mathrm{~dB}$.

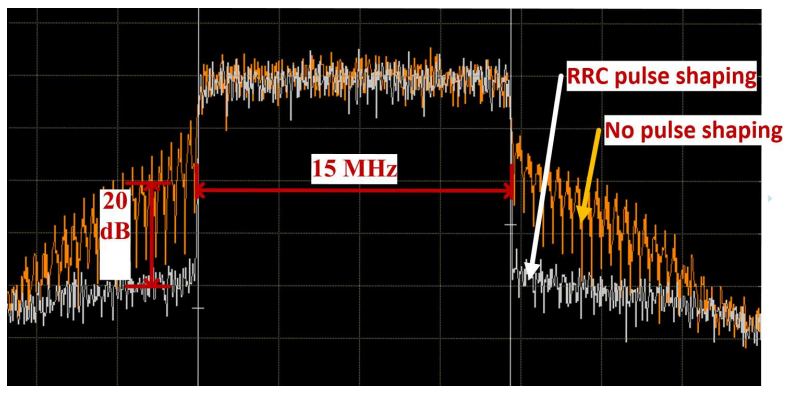

Figure 6. Measured spectra of SEFDM systems with two pulse shaping schemes. The center frequency is $2 \mathrm{GHz}$. 


\section{Measurement Results}

Four aggregation scenarios (A, B, C, D) are illustrated in Fig. 7 and used to study the impact of potential future 5G signals (SEFDM) on existing 4G signals. The 4G OFDM signal performance is evaluated via constellation diagram. The figure shows measurements of coexistent signal spectra and constellation of the $4 \mathrm{G}$ OFDM signal.

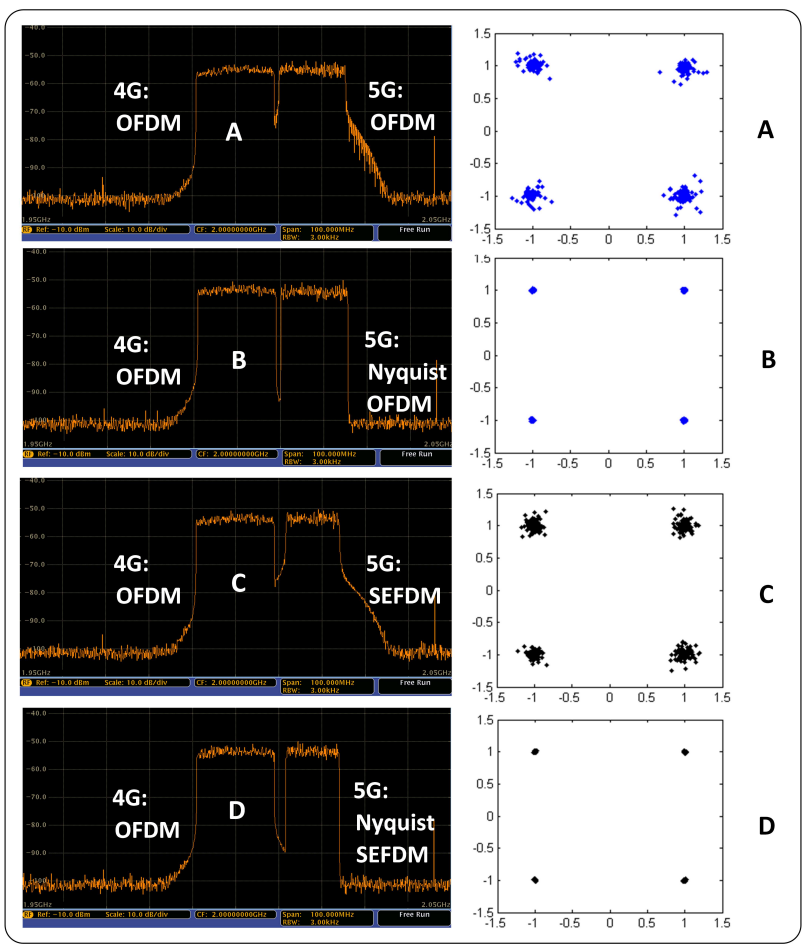

Figure 7. Four scenarios for $4 \mathrm{G}$ and $5 \mathrm{G}$ coexistent signals testing. The constellation diagrams on the right side are generated based on the 4G OFDM signal band in each scenario.

\section{- Scenario A:}

In this scenario, a potential future $5 \mathrm{G}$ signal is assumed to be OFDM. A $10 \%$ protection gap, which is equal to $1.5 \mathrm{MHz}$, between the two signal bands, is reserved. The spectra clearly shows the out-of-band power leakage of 5G OFDM signal. Constellation is the approach that judges the interference effect. In this comparison, we employe a bypass channel, which indicates no AWGN and no multipath effects. However, the constellation points are scattered, which is caused by the high outof-band power leakage from the $5 \mathrm{G}$ signal band.

\section{- Scenario B:}

The newly proposed Nyquist-OFDM signal is aggregated as the $5 \mathrm{G}$ candidate. The protection gap is still 1.5 MHz. The out-of-band power leakage of this signal is significantly reduced. Therefore, the adjacent channel interference is reduced. The constellation attached on the right shows the significantly improved performance.

\section{- Scenario C:}

This is the case that a typical SEFDM signal is designed for the $5 \mathrm{G}$ system. Due to the compressed bandwidth of SEFDM, the protection gap is beneficially increased to 3
MHz. It is evident that the out-of-band power is not flat, which is due to non-ideal analogue filters from the PXI. The reason to test SEFDM signal in this case is to show the saved bandwidth benefit. However, since no pulse shaping is used, the high out-of-band power leakage still exists resulting in scattered constellation.

\section{- Scenario D:}

In the last scenario, testing is for the newly proposed Nyquist-SEFDM signal, which compresses bandwidth and reduces out-of-band power leakage simultaneously. From the illustrated spectra, the protection gap between two signal bands is extended as expected from scenario C. In a future system design, due to this extra bandwidth, the signal band on the right may be shifted closer to the first signal band. Thus, the overall occupied bandwidth would be compressed. The reduced out-of-band power benefit of this scenario is demonstrated in the constellation diagram, which shows almost no interference.

\section{CONCLUSIONS}

This work aims to show the benefits of SEFDM waveform over OFDM via both simulation and experiment. The benefit has been clearly presented in the application scenario where more users can be serviced simultaneously with a more flexible channel allocation scheme. Via simulation, applying RRC pulse shaping in SEFDM is therefore beneficial in two aspects; it simplifies signal detection by reducing neighbour interference and improves spectral efficiency through bandwidth compression out-of-band power suppression. The coexistence experiment was carried out to test mutual interference of two closely packed signals. By using pulse shaping filters, the SEFDM signal has 20dB leakage power reduction. The four coexistence experiment showed that SEFDM has compressed bandwidth than typical OFDM and its pulse shaped waveform has neglected impact on legacy $4 \mathrm{G}$ signals.

\section{ACKNOWLEDGEMENT}

This work was supported in part by the Engineering and Physical Sciences Research Council "Impact Acceleration Discovery to Use" Award and in part by the EU Funded 5G Exchange Innovation Project 5GEx (www.5gex.eu).

\section{REFERENCES}

[1] 3GPP TS 36.300 version 8.12.0 Release 8, "Evolved universal terrestrial radio access (E-UTRA) and evolved universal terrestrial radio access network (E-UTRAN); overall description; stage 2 (release 8)," April 2010.

[2] 3GPP TR 36.913 v.10.0.0, "Requirements for further advancements for evolved universal terrestrial radio access (E-UTRA) (LTE-Advanced)," Rel. 10, Apr. 2011.

[3] J. Andrews, S. Buzzi, W. Choi, S. Hanly, A. Lozano, A. Soong, and J. Zhang, "What will 5G be?" IEEE Journal on Selected Areas in Communications, vol. 32, no. 6, pp. 1065-1082, June 2014.

[4] M. R. Palattella, M. Dohler, A. Grieco, G. Rizzo, J. Torsner, T. Engel, and L. Ladid, "Internet of things in the $5 \mathrm{G}$ era: Enablers, architecture, and business models," IEEE Journal on Selected Areas in Communications, vol. 34, no. 3, pp. 510-527, March 2016.

[5] T. Xu and I. Darwazeh, "Non-orthogonal narrowband internet of things: A design for saving bandwidth and doubling the number of connected devices," IEEE Internet of Things Journal, 10.1109/JIOT.2018.2825098 2018. 
[6] H. Shariatmadari, R. Ratasuk, S. Iraji, A. Laya, T. Taleb, R. Jntti, and A. Ghosh, "Machine-type communications: current status and future perspectives toward 5G systems," IEEE Communications Magazine, vol. 53, no. 9, pp. 10-17, September 2015.

[7] M. Rodrigues and I. Darwazeh, "A spectrally efficient frequency division multiplexing based communications system," in Proc. 8th Int. OFDM Workshop, Hamburg, 2003, pp. 48-49.

[8] Y. Feng and J. Bajcsy, "Information rate of multi-antenna spectrallyefficient FDM communication," in 2017 15th Canadian Workshop on Information Theory (CWIT), June 2017, pp. 1-5.

[9] T. Xu and I. Darwazeh, "Transmission experiment of bandwidth compressed carrier aggregation in a realistic fading channel," IEEE Transactions on Vehicular Technology, vol. 66, no. 5, pp. 4087-4097, May 2017.

[10] _ "Nyquist-SEFDM: pulse shaped multicarrier communication with sub-carrier spacing below the symbol rate," in 2016 10th International Symposium on Communication Systems, Networks and Digital Signal
Processing (CSNDSP), July 2016, pp. 1-6.

[11] T. Xu, S. Mikroulis, J. E. Mitchell, and I. Darwazeh, "Bandwidth compressed waveform for $60-\mathrm{GHz}$ millimeter-wave radio over fiber experiment," Journal of Lightwave Technology, vol. 34, no. 14, pp. 3458-3465, July 2016.

[12] H. Ghannam and I. Darwazeh, "Comparison of Turbo decoder and Turbo equalizer for spectrally efficient FDM system," in 2016 10th International Symposium on Communication Systems, Networks and Digital Signal Processing (CSNDSP), July 2016, pp. 1-6.

[13] T. Xu, "Bandwidth compressed waveform and system design for wireless and optical communications: Theory and practice," Ph.D. dissertation, University College London - Department of Electronic and Electrical Engineering, June 2017.

[14] H. Ghannam and I. Darwazeh, "SEFDM over satellite systems with advanced interference cancellation," IET Communications, vol. 12, no. 1, pp. 59-66, 2018. 